\title{
Multi-agent simulation modeling of online Internet discussions ${ }^{1}$
}

\author{
Eduard A. Babkin \\ Professor, Department of Information Systems and Technologies \\ National Research University Higher School of Economics \\ Address: 25/12, Bolshaya Pecherskaya Street, Nizhny Novgorod, 603155, Russian Federation \\ E-mail:eababkin@hse.ru
}

\section{Tatiana S. Babkina}

Senior Lecturer, Department of Information Systems and Technologies National Research University Higher School of Economics Address: 25/12, Bolshaya Pecherskaya Street, Nizhny Novgorod, 603155, Russian Federation E-mail: tbabkina@hse.ru

\section{Boris I. Ulitin}

Senior Lecturer, Department of Information Systems and Technologies National Research University Higher School of Economics Address: 25/12, Bolshaya Pecherskaya Street, Nizhny Novgorod, 603155, Russian Federation E-mail: bulitin@hse.ru

\begin{abstract}
Joint analysis of the general structure of online Internet discussions and different attributes of particular text comments becomes an important scientific task in theoretical and applied aspects. Although methods of machine learning facilitate stochastic analysis of text messages, appropriate modeling of dynamics of online discussion and psycho-linguistic characteristics of comments in the presence of multiple individual authors remains the unresolved problem. In this article, the authors suggest applying the methods of multi-agent simulations for resolution of that problem. This work offers two models of online discussion which allow us to take into account characteristics of individual comments and the presence of multiple authors with individual models of behavior. The behavior models are designed in the result of analysis of actual online Internet discussions. The first model is centralized and represents the behavior of each author in the same manner, using a set of fixed parameters. In comparison to the centralized model, the multi-agent distributed model states the individualized behavior for every author through the Markov chain of the special form. Such individualized structure allows us not only to approach the real dynamics of the discussion, but also to compare the models with the actual online Internet discussions. Using pre-processed factual data of real discussions from various Internet portals became an important feature of the suggested approach to simulation modeling. Pre-processing includes expert evaluation of psycho-linguistic characteristics (intent and content analysis), as well as methods of mathematical statistics. Therefore, this research is a positive example of inter-disciplinary research of Internet communication phenomena.
\end{abstract}

\footnotetext{
${ }^{1}$ This work was supported by the Russian Foundation for Basic Research (project No. 16-06-00184 A)
} 
Key words: communication models, online discussions, multi-agent systems, simulation modeling.

Citation: Babkin E.A., Babkina T.S., Ulitin B.I. (2018) Multi-agent simulation modeling of online Internet discussions. Business Informatics, no. 2 (44), pp. 17-29.

DOI: 10.17323/1998-0663.2018.2.17.29.

\section{Introduction}

A nalysis of the general structure of online Internet discussions and the content of particular text comments is becoming an important scientific task. In its fundamental aspects, studying the phenomenon of various forms of online discussions (blogs, chats, etc.) allows us to deepen our understanding of the phenomenon of interpersonal and network communications. The applied aspect of the problem, in turn, is related to the problems of creating intelligent advisory systems [1], increasing the effectiveness of electronic marketing and e-business [2], new forms of spontaneous and organized political activity $[3,4]$ and other significant sociological and economic problems.

The research task proposed in this article has a strong interdisciplinary flavor. Although methods of machine learning facilitate the automatic detection of emotional aspects of comments, detailed analysis of comments in the Internet discussions requires the development of both qualitative and quantitative methods of textual analysis based on a multiparameter peer review [5].

In this article, the authors propose two new multi-agent models of online discussion. The behavior of both models is designed in the result of analysis of actual online Internet discussions (media sites). The first model contains a centralized block for generating comments which is responsible for the behavior of agentauthors. In the second model, the behavior of agent-authors is individualized and defined through a multilevel hierarchical Markov chain.
Compared with other machine learning approaches to modeling discussions [3, 6-9], the proposed solutions provide new opportunities to model the behavior of individual participants in the discussion and to predict its development in general. The model structure created allows us not only to define such traditional discourse characteristics as intentions and the referential object of individual comments $[10,11]$, but also to generate the structure coincident with the actual discussions on the basic graph metrics, such as the central vertex/clique of the graph, the number of branches of the discussion, the average number and variance of the number of comments in the branches of the discussion. In addition, the use of a multi-agent paradigm allows us to individualize the behavior of the specific agent-author.

The article is structured as follows. In Section 1, the general aspects of the problem of online discussions analysis and prediction is described. Section 2 contains a definition of the structure and dynamics of the multiagent models created, software implementations of which are presented in Section 3. In Section 4, the validation of implementations created and their analysis are provided. We conclude the article by summarizing the results, comparing them with known analogues and setting specifications for future research.

\section{Main directions of online Internet discussions research}

In order to aalyze the appearance of different words and phrases, current studies of online 
Internet discussions use various types of statistical estimates based on the distribution of Dirichlet [12-14]. The most revealing examples of the research on our subject are the work by Wang et al. [8] and original methods for structure prediction by Yano, Cohen and Smith [3] and Ritter et al. [7]. In addition, the results of recent years in the wide research field of "tone analysis" $[2,9,15-17]$ can be interesting. For example, in the work [9], the authors provide an effective application of machine learning techniques using neural artificial networks.

However, despite the aforementioned multiple theoretical and practical results, some problems remain unresolved. Among such problems are the possibility of modeling individual comments on several parameters (referential object, intention) and modeling the consistent formation of the discussion structure.

In order to solve these problems, the interdisciplinary approach can be used. Such an approach should coherently combine both psycholinguistic methods of qualitative expert evaluation of texts and methods of modeling the behavior of individual entities.

Qualitative evaluation of the text comments of real online discussions by a group of independent experts makes it possible to form a set of empirical data necessary for preliminary statistical processing. In terms of interdisciplinary research, we organized the psycholinguistic expert processing of 300 articles of leading Russian Internet media (with user comments in an amount from 24 to 100 per article). As a result, generalized typologies of intentions, content (content-codes) and referential objects were developed. Subsequently, using these data, we formed the necessary categorical matrices and estimated the statistical reliability of the appearance of various combinations of intentions, content codes and referential objects in the discussions. A detailed description of this part of the research is beyond the scope of this article and can be found with information about the methodology developed, typologies, results and analysis in $[4,5]$.

Individual based simulations [18] naturally complement the results of qualitative expert evaluation of online discussions and allow us to construct a class of reproducible computer models that describe the behavior of individual authors, as well as the structure of the discussion in general. Multi-agent programming systems, which are widely used for modeling various social phenomena $[19,20]$ and optimization [21, 22], can be a practical tool for implementing such models.

\section{Proposed multi-agent models of discussion}

In the proposed multi-agent models, we represent the structure of the discussion as a directed tree graph, in which the original article is the root, and the comments become leaves of the tree.

Each vertex of the graph is described with a certain set of parameters: a set of intentions, a content, and a referential object. Such a mathematical structure is used in both a centralized and a distributed model. The difference is only the mechanism for generating new vertices of the discussion graph.

\subsection{Description of the structure of the model in terms of a multi-agent system}

Both models contain two agent types. The first type is active (intelligent) agent-authors (authors), which have its own behavior and can make decisions. Active agents create the second type agents-comments (comments), which is passive and does not have its own behavior.

Each agent-author (Figure 1) is characterized by a unique identifier and is able to produce comments according to a set of global varia- 


\begin{tabular}{|c|c|}
\hline Autor & Comment \\
\hline - id : int & \multirow{3}{*}{$\begin{array}{l}\text { - id : int } \\
\text { - intent : string } \\
\text { - reference0bject : int } \\
\text { - content : int } \\
\text { - author_id : int } \\
\text { - is_root : boolean }\end{array}$} \\
\hline \multirow{3}{*}{$\begin{array}{l}\text { + read_parametrs_from_file() : void } \\
\text { + generate-comment() : void } \\
\text { - calculate_intent() : string } \\
\text { - choose_needed-way }() \text { : int }\end{array}$} & \\
\hline & \\
\hline & + find-all-pairs() : void \\
\hline
\end{tabular}

Fig. 1. UML-representation of models' agents

bles: ways, which stores all possible paths from the root to the leaf vertices of the branches of the discussion tree, and neededwayid and cur_ way, that store the branch of the discussion, with which the agent-author works at the current stage of the model.

In turn, agent-comments are described with only their own attributes: intention (intent), referential object (referenceObject), content (content), identifier of the agent-author generated the current comment (author_id), and flag (is_ root), which allows us to distinguish the original article from the comments relating to it.

Also, there are several environment variables in every model required for its statistical analysis: branches_count, which stores the number of branches of the discussion tree, comments count_in_branches_Mand comments_count_ in_branches_D, that store the average number and standard deviation of the number of comments in all branches of the discussion tree respectively, etc.

\subsection{Description of agent behavior rules}

The behavior of agent authors in the model is specified by a set of rules. The need to apply each specific rule is determined at each step of the model and depends on the values of the environment variables and the state of the discussion tree.

In total there are three rules: comment generation rule, comment characteristics definition rule, the rule of the location depth of the comment and branch comment selection rule. The first two rules are the same for both multiagent and centralized models, while the rest depend on the version of the model.

The comment generation rule is:

$$
I g c=\left\{\begin{array}{l}
1, \text { if } x<C g c_{i}^{t} \\
0, \text { else }
\end{array},\right.
$$

where $I g c$ - indicator, responsible for comment generation;

$x$ - value of a uniformly distributed random variable $X(0,1)$;

$i=1,2, \ldots, I-$ identifier of the current agentauthor;

$C g c_{i}^{t}$ - threshold value determined by the behavior model of the agent-author $i$ at time $t$.

It is important to note that in the centralized model the behavior of agent-authors is the same and does not change over time, i.e. $C g c_{i}^{t+1}=C g c_{i}^{t}=C g c_{1}^{0}, \forall i \geq 1$ and $t \geq 0$.

\section{The comment characteristics definition rule} is defined according to the information about the pair rules for generating comments, each of which has the form:

$$
i_{1} r_{1} \rightarrow i_{2} r_{2} N
$$

where $i_{1} r_{1}$ and $i_{2} r_{2}-$ intention and referential object of parent and child comment respectively;

$N$ - the quantity that a given pair of parent and child comments met in the discussion tree.

By grouping a family of such rules according to the parent comment, we get for each of them the following set of pair rules: 


$$
i_{p} r_{p} \rightarrow\left\{i_{j} r_{j} N_{j}\right\},
$$

where $\left\{i_{j} r_{j} N_{j}\right\}, j=1,2, \ldots, J$ contains all child comments related to the current parent comment $i_{p} r p$.

At this:

$$
j=\left\{\begin{array}{c}
1, \text { else } 0 \leq x<N_{1} \\
2, \text { else } N_{1} \leq x<N_{2} \\
\cdots \\
J, \text { else } N_{J-1} \leq x<N_{J},
\end{array}\right.
$$

where $x$ - value of a uniformly distributed random variable $X\left(0, \sum_{j} N_{j}\right)$.

It is important to note that in the centralized model the set of these pair rules is the same for all agent-authors in contrast to the multi-agent model, where these pair rules are individualized for every agent-author according to its behavior model at time $t$.

The rule of the location depth of the comment in the centralized model is identified as follows:

$$
\text { Iclt }=\left\{\begin{array}{l}
1, \text { if } x<C l t \\
0, \text { else }
\end{array},\right.
$$

and in the case of the multi-agent model it is generalized in the next form:

$$
\text { Imlt }=\left\{\begin{array}{l}
1, \text { if } x<C t_{i}^{t} \\
2, \text { if } C t_{i}^{t} \leq x<C n t_{i}^{t} \\
3, \text { if } C n t_{i}^{t} \leq x<C m_{i}^{t}, \\
4, \text { if } \mathrm{Cm}_{i}^{t} \leq x<C n l_{i}^{t} \\
5, \text { else }
\end{array}\right.
$$

where Iclt - indicator identifying the location depth of the comment in the centralized model ( 1 - comment is generated to the leaf vertices of the discussion tree, otherwise - to non-leaf vertices);

Ilmt - indicator identifying the location depth of the comment in the multi-agent model $(1-$ comment is generated to the root vertices of the discussion tree, 2 - to a depth of not more than $M$ from the root vertices, 3 to a depth of more than $M$ from the root but not more than $M$ of leaf vertices, $4-$ to a depth of not more than $M$ from leaf vertices, 5 - to the leaf vertices of the discussion tree);

$x$ - value of a uniformly distributed random variable $X(0,1)$;

$i=1,2, \ldots, I-$ identifier of the current agentauthor;

$C l t$ - threshold value determined by the value of environmental variable leaf_top_connection_probability;

$M$ - value that characterizes the location depth of the comment;

$C t_{i}^{t}, \mathrm{Cnt}_{i}^{t}, \mathrm{Cm}_{i}^{t}, \mathrm{Cnl}_{i}^{t}$ - threshold values that describe quantitative distribution of the agentauthor $i$ comments on the depth of the discussion branch at the time $t$ and depend on the behavior model of the corresponding agentauthor.

Branch comment selection rule is based on the configuration of the discussion tree at a particular time $t$. Let there be branches in the discussion tree, the length of each of them is $L_{i}(1 \leq i \leq M, i \in \mathbb{N})$. The agent-author $A$ generated $c c_{i}$ number of comments in branch $i$ $(1 \leq i \leq M, i \in \mathbb{N})$. Then in the centralized model the rule for selection the branch $i$ for comment generation can be formalized as follows:

$$
i=\left\{\begin{array}{c}
1, \text { if } 0 \leq x<c c_{1} \\
2, \text { if } c c_{1} \leq x<c c_{2} \\
\cdots \\
M, \text { if } c c_{M-1} \leq x<c c_{M}
\end{array}\right.
$$

and in the case of the multi-agent model is generalized in the next form:

$$
i \in I=\left\{\begin{array}{l}
\text { Io, if } 0 \leq x<C o_{A}^{t} \\
\text { If, if } C o_{i}^{t} \leq x<C f_{A}^{t} \\
\text { Imf, if } C f_{i}^{t} \leq x<C m f_{A}^{t} \\
\text { Im, else }
\end{array},\right.
$$

where $I o=\left\{i: c c_{i}=0\right\}, I f=\left\{i: \frac{c c_{i}}{L_{i}}<S\right\}$,

$\operatorname{Imf}=\left\{i: S<\frac{c c_{i}}{L_{i}}<1-S\right\}, \operatorname{Im}=\left\{i: \frac{c c_{i}}{L_{i}}>S\right\}$

$x$ - value of a uniformly distributed random 
variable $X\left(0, \sum_{j} c c_{j}\right)$;

$S-$ a threshold value;

$C o_{A}^{t}, C f_{A}^{t}, C m f_{A}^{t}-$ threshold values describe quantitative distribution of the agent-author $A$ comments on the branches of the discussion tree at time $t$ and depend on the behavior model of the corresponding agent-author. In

both cases, the selected branch and comments on it are stored in the variables neededwayid and cur_way respectively.

As a result, we can use this set of rules in order to wholly describe the behavior model of every agent-author in the case of the centralized model (Figure 2).

In contrast to a centralized model, in a dis-

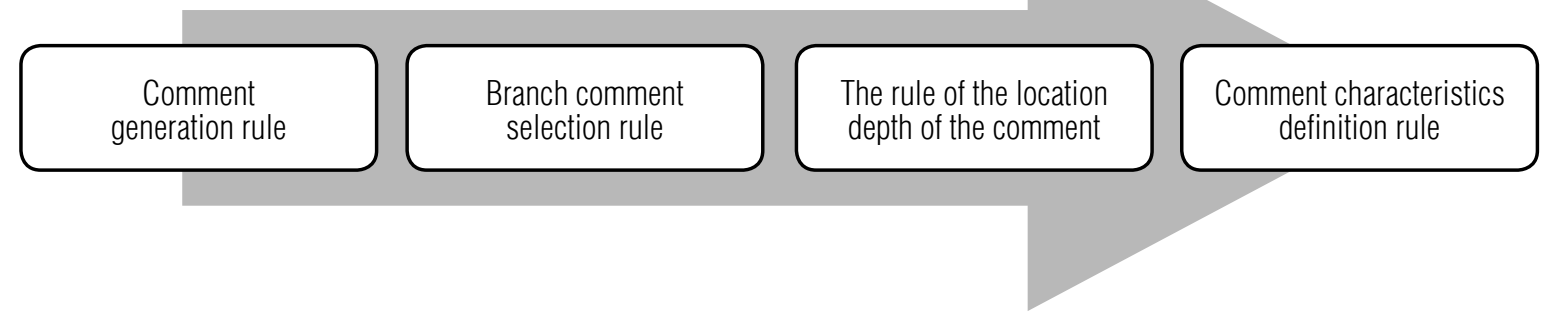

Fig. 2. A conceptual generalized scheme of behavior of an agent-author

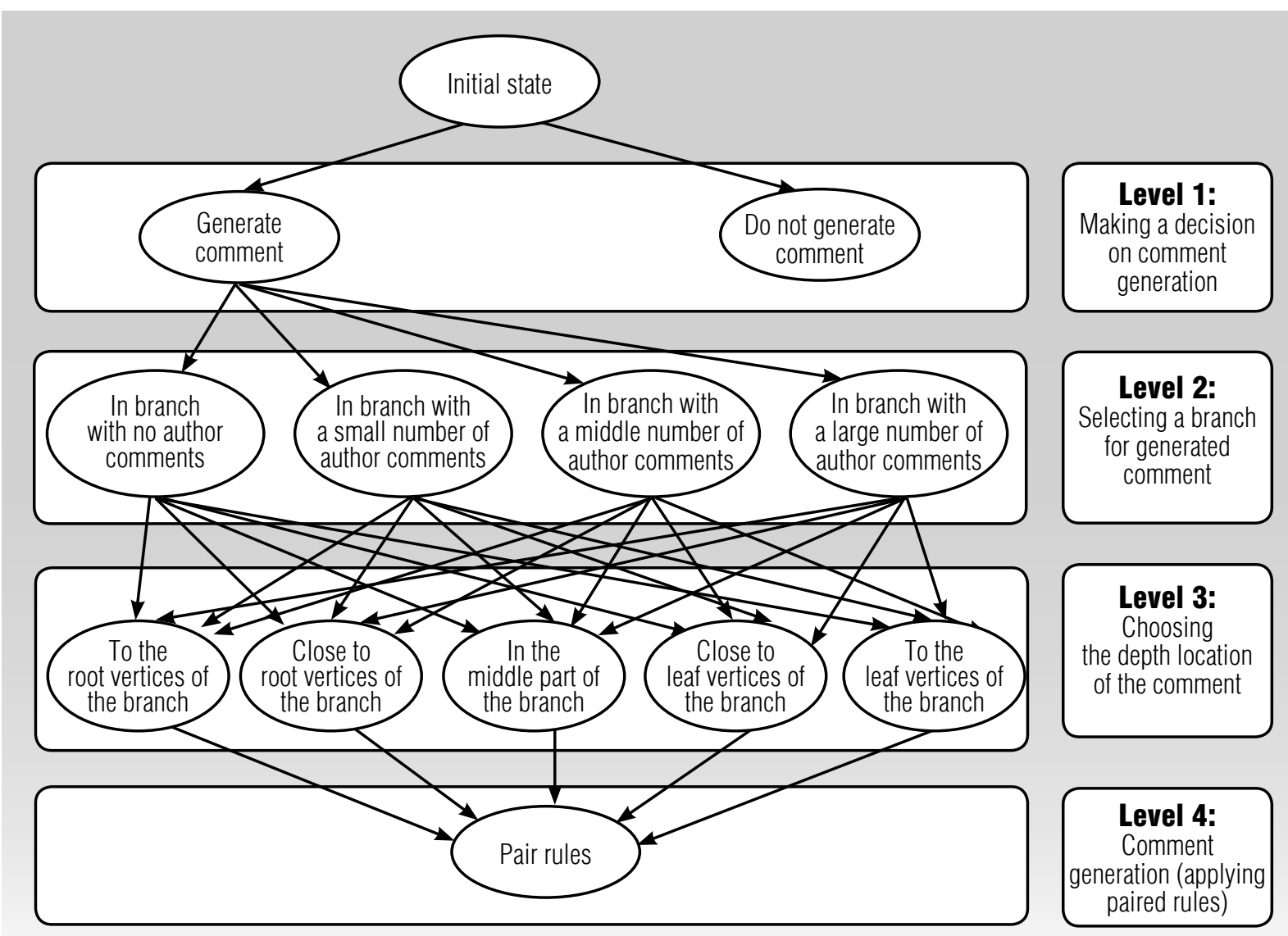

Fig. 3. The conceptual scheme of the agent-author behavior Markov chain 
tributed multi-agent model, agent-author behavior becomes more complex and is individualized for each agent-author through the hierarchical Markov chain [23] (Figure 3). At the same time, the stages (levels of the Markov chain) of this model coincide with the centralized model.

It is also important that we receive a set of Markov chains for agent-authors as an output of the centralized model work and can use them as an input for the distributed multiagent model. This interrelated organization of the work of both models allows us to conduct the subsequent comparative analysis of their identity.

\section{Program implementation of the simulation system}

For program implementation of the proposed discussion models, we used the multi-agent simulation environment NetLogo (v.6.0.1+) [24]. In addition to this, we have developed a Java program module using an open-source JUNG library (v.2.0.1+) [25] in order to visualize and analyze graphs of Markov chains which describe the individualized authoragents behavior.

The key point of centralized model program implementation is that it does not require any input parameters, and at the output periodically generates pairs of files with description of the author-agents behavior. Examples of such files are represented in Figure 4 (hereinafter the codes represent our intent classification).

As we mentioned above, these centralized model output files are input for the distributed multi-agent model implementation, which, in turn, also generates similar files as its output. Such a mechanism allows to compare the results of the work of two models and analyze their identity. In order to organize such a comparison, we transfer all these files to the input of the Java module, which extracts and visualizes the paired rules contained in them (Figure 5).

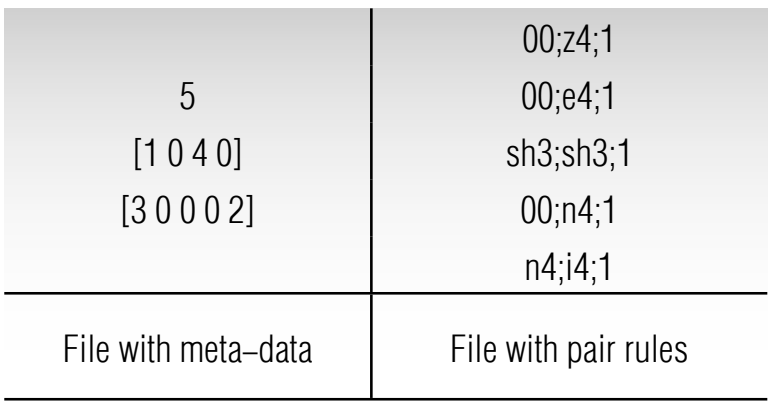

Fig. 4. Examples of centralized model output files (the first line contains the total number of author's comments, the two others their depth location distribution and distribution on the branches of the discussion tree respectively)

In order to demonstrate changes in the author-agent Markov chain over time, the visualization contains in the center all parentcomments, which appeared throughout the work model, while different frames (clusters) of the scheme combine child-comments, that arose at different stages of the model work.

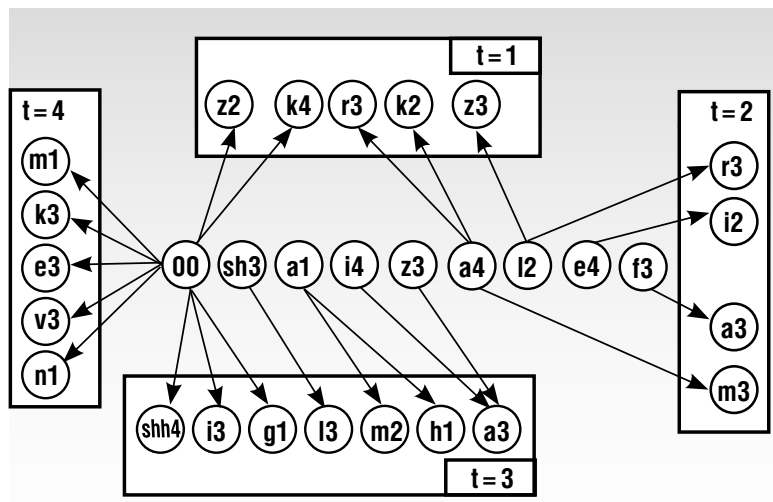

Fig. 5. Examples of visualization of the output graph of the author-agent Markov chain (circles mean comments (coded by intension and referential object), rectangles frame comments generated by the author at each time period of the model work)

\section{Analysis of the experiment results}

In order to evaluate the quality of the models developed and their correspondence with the real online discussions graphs, we conducted several series of experiments for the 

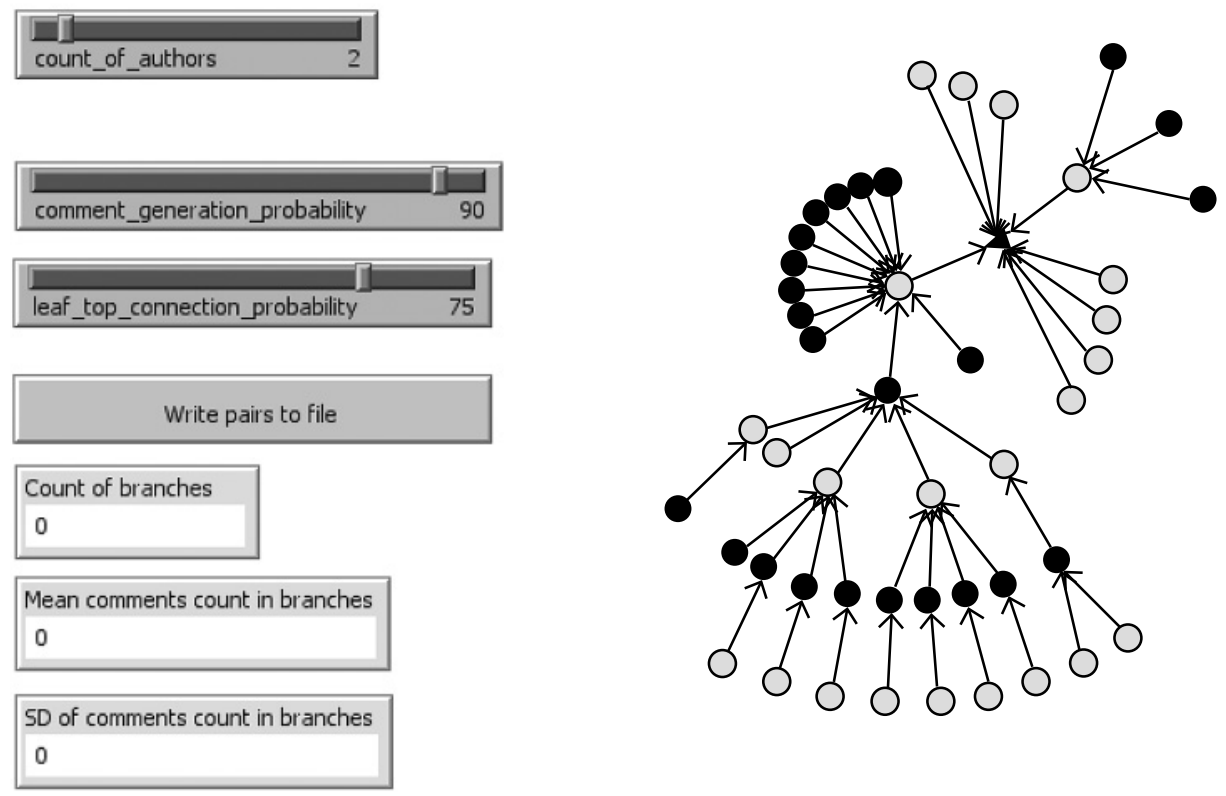

Fig. 6. The initial parameters of the control panel and the final state

of the discussion graph of the centralized model (different colors mark comments generated by different authors)

centralized and multi-agent versions of the model, respectively. We considered the experiment at small dimensions (the number of comments $\sim 25$ ) as well as on large dimensions (the number of comments $\sim 200$ ) of the discussion. Such values for comment numbers were defined according to the sizes of real online discussion trees (see Section 1) that allow us in what follows to compare the results of model works with real factual data.

First, we conducted an experiment with a small discussion (Figure 6). For this we defined two author-agents. At each model stage, these agents generate a comment with probability 0.9 and connect the generated comment to the not leaf and leaf vertices with probabilities 0.25 and 0.75 , respectively. The time period for the model work is 25 ticks, and result saving occurs every 5 ticks.

Then, based on the results of the centralized model, we launched the distributed version (the graph of the final state is represented in Figure 7) and compared the final discussion trees by their main indicators with each other.

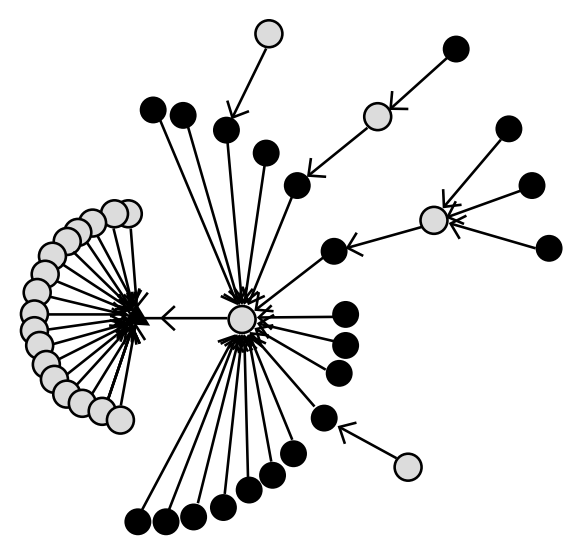

Fig. 7. The final state of the discussion graph of the distributed model

As we can see, visualizations of these graphs are similar (Figures 6 and 7). For example, most of the comments center around the original article (vertex $\# 0$ ) and one of the childcomments relating to it (vertex/comment \#3). These vertices are central for the discussion tree by the number of input/output vertices: root vertex has degree 18 in the first case and 9 in the second one, as well as vertex/comment 
$\# 3-17$ and 11 in the first and the second cases respectively.

Now, in order to approve the correspondence of results of the centralized and multi-agent models and real discussions trees, we should provide detailed analysis of the author-agents behavior through comparison of the Markov chain pair rules.

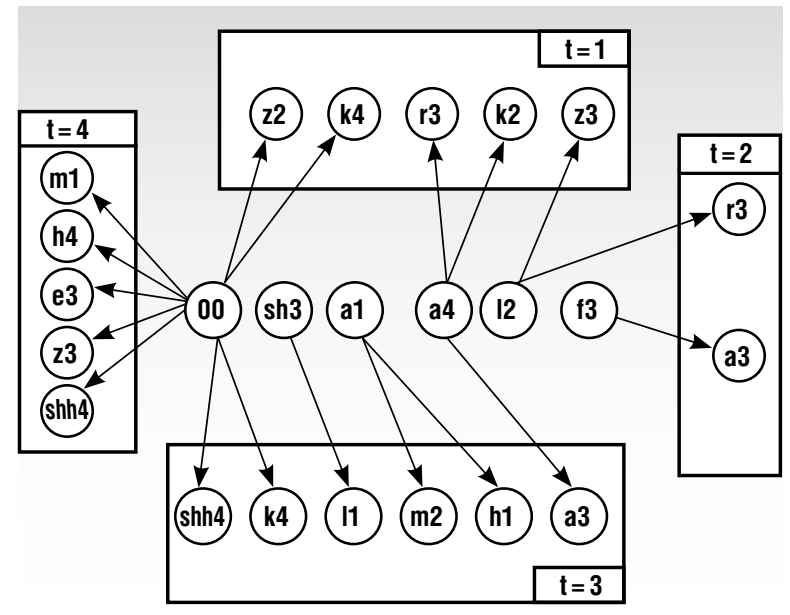

Fig. 8. The first author-agent Markov chain after the work of the centralized model
For the first author-agent this Markov chain was represented above (Figure 5). Comparing it with the model for the same author, obtained during the work of the centralized model (Figure 8), we find that the central part of both schemes contains many identical vertices. Such a clear similarity testifies to the preservation of the general principles of the author's behavior in both the individualized and the centralized models. Analysis of the behavior of the second author participating in the models demonstrates similar results.

After the analysis of small discussions, we should provide a similar analysis of the large discussions. For this we fix the initial parameters of the centralized model as in the previous case (Figure 6) but increase the duration to 230 ticks. As a result, at the output, we obtain the following Markov chain of the first agentauthor behavior (Figure 9).

Then we transfer the Markov chain so obtained to the input of the distributed multi-agent model, which also produces a Markov chain of the first author-agent behavior as the output (Figure 10).

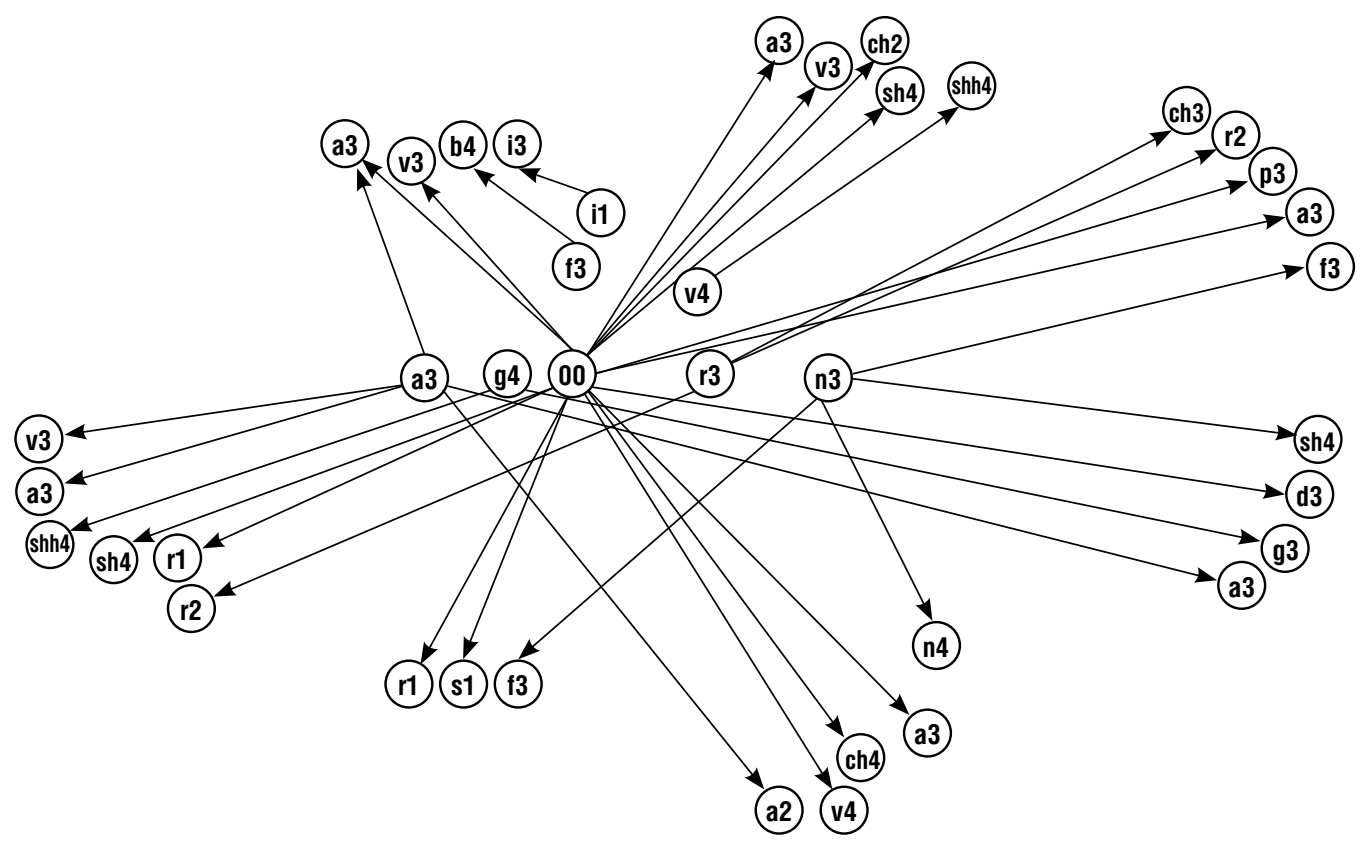

Fig. 9. Fragment of the first author Markov chain after the run of the centralized model (230 ticks) 


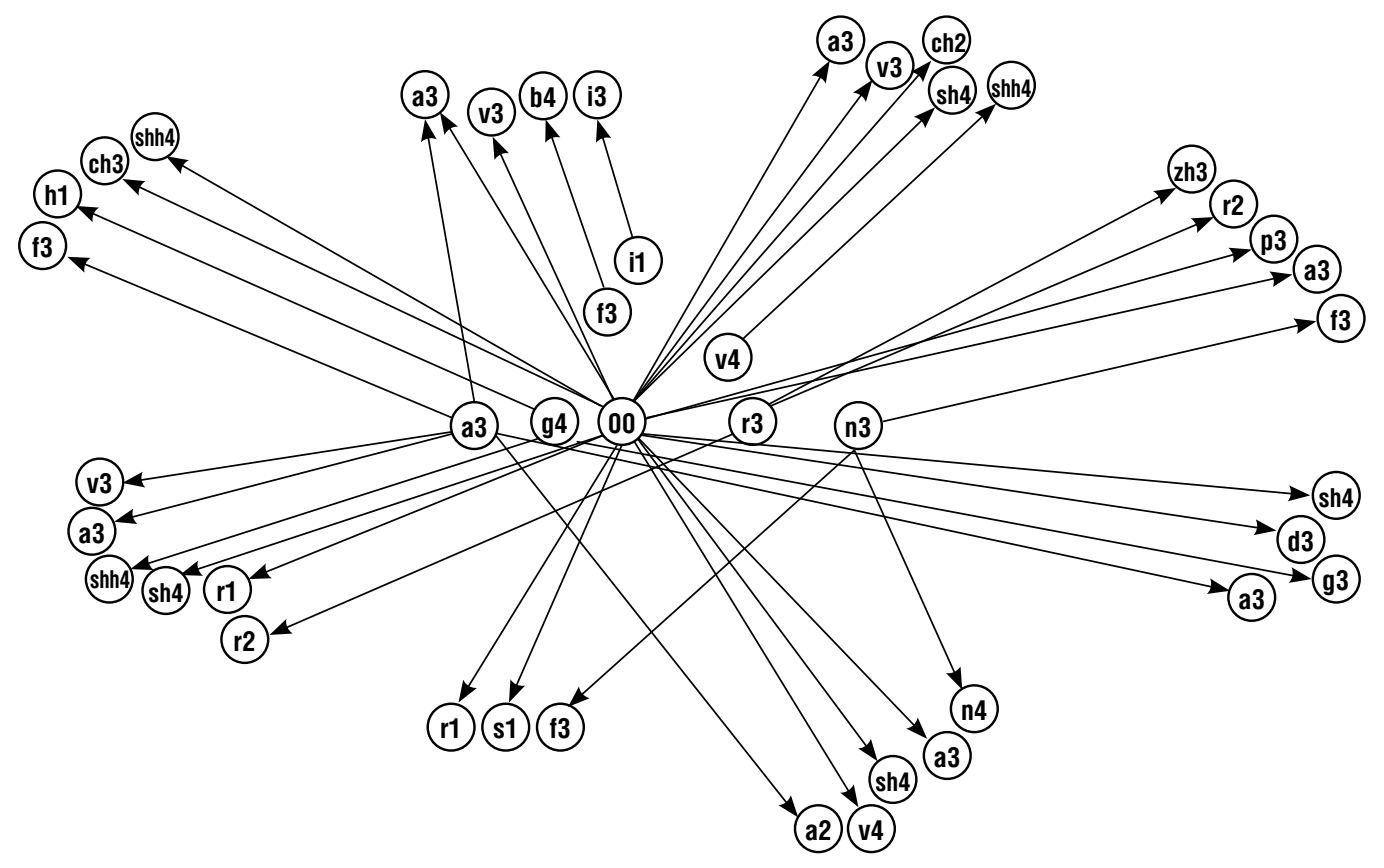

Fig. 10. Fragment of the first author Markov chain after the run of the multi-agent model (230 ticks)

Comparing these two schemes of the first author-agent behavior, we can find several similarities. For example, the central clique in both graphs includes vertices 00 (original article) and comments with intentions a3, r3, g4, etc. In addition, the similarity in intentions is also revealed when comparing the corresponding segments of Markov chains, all of which indicates the identity of the author's behavior in the models. Confirmation of this can also be found from comparison of files with meta-data on the hierarchical Markov chain of the author's behavior (Figure 11).

\begin{tabular}{l|l}
296 & 326 \\
{$[427511554]$} & {$[828710354]$} \\
{$[6736376670]$} & {$[7736776670]$} \\
\hline Individualized & General \\
(distributed) model & (centralized) model \\
\hline
\end{tabular}

Fig. 11. Metadata files of the first author after the work of models
During the distributed model run, the first author-agent generated 296 comments. 42 of which are in branches with no comments by the corresponding author, 115 - in branches with a middling number $(20-80 \%$ of the total in the branch) of comments by the corresponding author and $75-$ in branches with a small number $(<20 \%$ of the total in the branch) of comments by the corresponding author. Other comments correspond to branches with a large number ( $>80 \%$ of the total in the branch) of comments by the corresponding author. 67 of these comments are connected to the root of the discussion tree, 70 - in leaf vertices, $66-$ closer to the discussion leaf vertices, and the rest - in the middle part of the discussion tree branch.

Furthermore, since the centralized model is based on data about paired rules from real online discussions, we can confirm a correspondence between the centralized model and the real discussion graphs. This can also be confirmed by comparing the Markov chain of a centralized model (Figure 9) with the Markov chain of real discussion (Figure 12). 


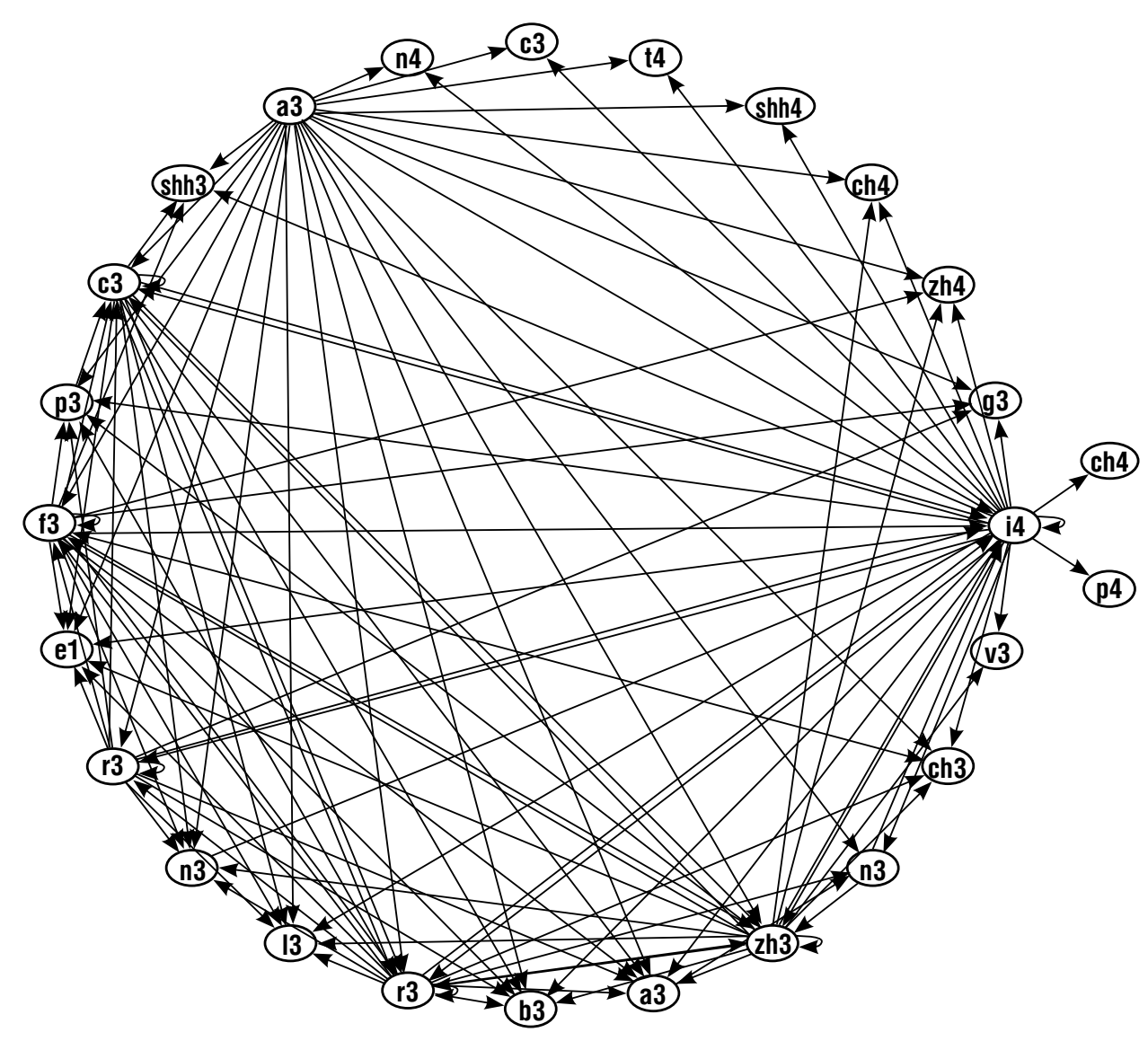

Fig. 12. Fragment of the agent-author Markov chain of a real discussion

These facts show that the models developed do not contradict the data of real online discussions and, therefore, can be used to analyze and predict their dynamics. In addition, since in the centralized model the author's behavior depends only on its previous actions and the state of the discussion graph to the present moment, a finite state machine model with memory can be used to model the author's behavior.

\section{Conclusion}

In this paper, we analyzed the behavior of authors during online discussions and problems which appeared through modeling of this process. A special feature of the research task was the combination of a quantitative evaluation of a large amount of factual data from electronic media and subsequent multi-agent modeling of the structure and dynamics of the discussion in terms of a stochastic multi-agent system using hierarchical Markov chains of a special kind. For this, we implemented two types of models which were validated by comparative analysis of the characteristics of the discussion trees.

It can be concluded that the rules developed for author-agents behavior appropriately reflect the basic principles of the discussion structure in general, as well as the characteristics of individual comments (intention, content, referential object). Key characteristics of discussion trees obtained from two models, coincide with each other and with the real online Internet discussions. These facts allow us to state that the models created can be used as an effective tool for predicting the dynamics of online discus- 
sions for a wide range of practical problems in the field of Internet marketing, political science and e-business.

In comparison with other studies in the field of modeling the discussion (in particular [6, $7])$, the proposed approach allows us to generate the discussion structure subsequently by a group of authors in the form of a graph with such important characteristics of individual comments as intention, reference object, content code.

In future research, we are going to pay special attention to the modification of proposed models needed to confirm the hypothesis that the author-agent behavior model can be suitably represented in the form of a finite automaton with memory. To achieve this goal, the model created can be transformed according to the classical "belief-desire-intention" (BDI) paradigm [26], where a finite automaton is responsible for "beliefs", and "desiresintentions" are generated by a stochastic module.

Unfortunately, the models proposed allow us to analyze only the intent aspect of the discussion, irrespective of the subject area in which this discussion is conducted. In order to resolve this limitation, a supplementary content analysis implementation to the model is required. This addition to the model can allow us to organize a more flexible configuration of the model, resulting in a study of the limitations on its application in different subject areas.

\section{References}

1. Yang D., Huang C., Wang M. (2017) A social recommender system by combining social network and sentiment similarity: A case study of healthcare. Journal of Information Science, vol. 43, no. 5, pp. 635-648.

2. Park K., Kim J., Park J., Cha M., Nam J., Yoon S., Rhim S. (2015) Mining the minds of customers from online chat logs. Proceedings of the 24th ACM International on Conference on Information and Knowledge Management (CIKM 2015), Melbourne, Australia, 19-23 October 2015, pp. 1879-1882.

3. Yano T., Cohen W.W., Smith N.A. (2009) Predicting response to political blog posts with topic models. Proceedings of the 2009 Annual Conference of the North American Chapter of the Association for Computational Linguistics: Human Language Technologies (NAACL HLT 2009), Boulder, Colorado, 1 May - 5 June 2009, pp. 477-485.

4. Kudryavtseva E.N., Radina N.K. (2017) Antisotsial'noe povedenie v online-diskussiyah [Antisocial behavior in online discussions] // Proceedings of the 3rd International Scientific and Practical Conference “Actual problems of mass consciousness research”, Penza, Russia, 24-25 March 2017, pp. 86-89 (in Russian).

5. Radina N.K. (2016) Intent-analiz onlayn-diskussiy (na primere kommentirovaniya materialov internet-portala InoSMI.ru) [Intention analysis of online discussions (based on the example of comments on the materials of the Internet portal InoSMI.ru)]. Mediascope, no. 4, p. 25 (in Russian).

6. Hoang T.A., Lim E.P. (2017) Modeling topics and behavior of microbloggers: An integrated approach. ACM Transactions on Intelligent Systems and Technology, vol. 8, no. 3, p. 44.

7. Ritter A., Cherry C., Dolan B. (2010) Unsupervised modeling of Twitter conversations // Human Language Technologies. Proceedings of the 2010 Annual Conference of the North American Chapter of the Association for Computational Linguistics: Human Language Technologies (NAACL HLT 2010), Los Angeles, California, 1-6 June 2010, pp. 172-180.

8. Wang J., Yu C.T., Yu P.S., Liu B., Meng W. (2015) Diversionary comments under blog posts. ACM Transactions on the Web, vol. 9, no. 4, p. 18.

9. Karpov N., Demidovskij A., Malafeev A. (2017) Development of a model to predict intention using deep learning. Proceedings of the 6th International Conference "Analysis of Images, Social Networks and Texts" (AIST 2017), Moscow, Russia, 27-29 July 2017; Lecture Notes in Computer Science, Revised Selected Papers. Springer, pp. 69-78. 
10. Kibrik A.A., Dobrov G.B., Zalmanov D.A., Linnik A.S., Lukashevich N.V. (2010) Referentsial'nyy vybor kak mnogofaktornyy veroyatnostnyy protsess [Referential choice as a multi-factor stochastic process]. Computer linguistic and intelligent technologies (according to "Dialog 2010" International Conference), vol. 9, no. 16. Moscow: RSUH, pp. 173-180 (in Russian).

11. Suleymanova E.A. (2012) O referentsial'nyh aspektah zadachi izvlecheniya faktov [On the referential aspects of the facts extracting task]. Program Systems: Theory and Applications, vol. 3, no. 3, pp. 41-56 (in Russian).

12. Blei D.M., Ng A.Y., Jordan M.I. (2003) Latent Dirichlet allocation. Journal of Machine Learning Research, no. 3, pp. 993-1022.

13. Jelodar H., Wang Y., Yuan C., Feng X. (2017) Latent Dirichlet Allocation (LDA) and topic modeling: models, applications, a survey. arXiv preprint, arXiv:1711.04305. 2017.

14. Paisley J., Wang C., Blei D.M., Jordan M.I. (2015) Nested hierarchical Dirichlet processes. IEEE Transactions on Pattern Analysis and Machine Intelligence, vol. 37, no. 2, pp. 256-270.

15. Balazs J.A., Velásquez J.D. (2016) Opinion mining and information fusion: A survey. Information Fusion, vol. 27, pp. 95-110.

16. Bele N., Panigrahi P.K., Srivastava S.K. (2017) Political sentiment mining: A new age intelligence tool for business strategy formulation. International Journal of Business Intelligence Research, vol. 8, no. 1, pp. 55-70.

17. Oleshkov M.Y. (2006) Modelirovanie kommunikativnogo protsessa [Simulation of the communication process]. Nizhny Tagil. NTSSPI (in Russian).

18. Macal C.M. (2016) Everything you need to know about agent-based modelling and simulation. Journal of Simulation, vol. 10, no. 2, pp. 144-156.

19. Garcia D., Garas A., Schweitzer F. (2017) An agent-based modeling framework for online collective emotions. Cyberemotions (eds. J. Holyst). Cham: Springer, pp. 187-206.

20. Sun J., Tang J. (2011) A survey of models and algorithms for social influence analysis. Social network data analytics (eds. C. Aggarwal). Boston, MA: Springer, pp. 177-214.

21. Babkin E., Abdulrab H., Babkina T. (2009) AgentTime: A distributed multi-agent software system for university's timetabling. From system complexity to emergent properties (eds. M.A. Aziz-Alaoui, C. Bertelle). Berlin, Heidelberg: Springer, pp. 341-354.

22. Satunin S., Babkin E. (2014) A multi-agent approach to intelligent transportation systems modeling with combinatorial auctions. Expert Systems with Applications, vol. 41, no. 15, pp. 6622-6633.

23. Ausin M.C. (2015) Markov chain Monte Carlo, Introduction. Wiley StatsRef: Statistics Reference Online. Available at: https://www.sciencedirect.com/science/article/pii/B0080430767004691 (accessed 02 April 2018).

24. Sklar E. (2007) Software review: NetLogo, a multi-agent simulation environment. Artificial Life, vol. 13, no. 2, pp. 1-9.

25. Yan-Biao B. (2004) The JUNG (Java Universal Network/Graph) Framework. Technical Report UCI-ICS 03-17. Irvine, CA: University of California. Available at: http://www.datalab.uci.edu/papers/JUNG_ tech_report.html (accessed 02 April 2018).

26. Fasli M. (2003) Interrelations between the BDI primitives: Towards heterogeneous agents. Cognitive Systems Research, vol. 4, no. 1, pp. 1-22. 